\title{
Widths of autoionizing states of He from a variational calculation using complex coordinates
}

\author{
C. A. Nicolaides and E. Adamides \\ Theoretical and Physical Chemistry Institute, National Hellenic Research Foundation, \\ 48 Vassileos Constantinou Avenue, Athens 501/1, Greece
}

(Received 6 August 1982)

\begin{abstract}
Using correlated complex functions with Gamow orbitals and a recently developed variational procedure, we have calculated the autoionizing widths of the $\mathrm{He} 2 s 2 p^{1} P^{\circ}, 2 p^{2}{ }^{\mathrm{I}} \mathrm{D}$, and $2 s^{2}{ }^{\mathrm{I}} S$ resonances. They are found to be in good agreement with previous accurate calculations which employed the golden-rule formula with real scattering functions.
\end{abstract}

\section{INTRODUCTION}

The complex energies of resonances can be obtained from schemes which use complex coordinates either in the Hamiltonian or in the wave function. ${ }^{1,2}$ For many-electron atoms, there are two basic problems associated with this approach: Firstly, a suitable many-body theory should be established which avoids the diagonalization of huge complex matrices while at the same time allows for the computation of partial widths. Secondly, since there is no Rayleigh-Ritz minimum principle, new variational procedures should be discovered which allow for the optimization of the trial functions.

In this report we present the first results of calculations on the $\operatorname{He} 2 s 2 p^{1} P^{\circ}, 2 p^{21} D$, and $2 s^{2}{ }^{1} S$ resonances which employ the correlated wave functions and theory of resonances and computational procedures which were developed in Refs. 2-5 having the aforementioned two problems as targets.

\section{CHOICE OF THE RESONANCE FUNCTIONS}

The form of the resonance wave function of complex coordinates $\rho=r e^{i \theta}$ is $^{2,3}$

$$
\psi(\rho)=a(\theta) \psi_{0}(\rho)+b(\theta) \chi(\rho),
$$

where $\psi_{0}$ is the part containing the localized analytic Hartree-Fock, and properly projected ${ }^{3}$ single and pair functions, and $\chi$ is the part containing the asymptotic pair correlation. For He, this pair function is given by

$$
\Pi_{2 s}\left(\rho_{1}, \rho_{2}\right)=A\left[1 s\left(\rho_{1}\right) g_{2 s}\left(\rho_{2}\right)\right],
$$

where $1 s(\rho)$ is the rotated hydrogenic $1 s$ orbital and $g_{2 s}(\rho)$ is the Gamow orbital ${ }^{2,3}$ whose radial part is expressed as

$$
g(r)=u(r)+f(r) r^{1+i \mu} e^{i k r},
$$

with

$$
u(r)=r^{l+i} e^{-\zeta r}, \quad f(r)=r^{-1}-r^{-1} e^{-d r} \sum_{n=0}^{l} \frac{(d r)^{n}}{n !} .
$$

$l$ is the angular momentum of the outgoing electron. The reasons for introducing the Gamow orbitals were given in Refs. $2-4$.

\section{OPTIMIZATION OF THE TRIAL FUNCTIONS}

The aim of this work was to test the validity of the variational principle suggested in Refs. 2-4 which is based on the minimization of the quantity

$$
\left|\frac{b^{2}}{a^{2}}-\frac{\Gamma}{|k|}\right|=\min ,
$$

where $a, b$ are the complex coefficients of Eq. (1) and $\Gamma,|k|$ are the calculated resonance width and energy. The first application of this principle was reported for the He $2 s 2 p^{1} P^{\circ}$ width using only a Hartree-Fock (HF) function. ${ }^{3}$ Here, the localized part $\psi_{0}$ contains electron correlation as well, obtained once for real coordinates, according to the theory of Refs. 2 and 3.

We did not insist on an extremely accurate $\psi_{0}$. Instead, we chose only those correlation vectors which included single and double excitations ( 6 configurations for ${ }^{1} P \circ, 11$ for ${ }^{1} D$, and 15 for ${ }^{1} S$ ) that are expected to correct the HF function the most. The HF function for the $2 s 2 p^{1} P{ }^{\circ}$ state was expanded in terms of three $s$ and two Slater $p$-type orbitals.

TABLE I. Widths of $2 s^{2} 2 p^{1} P^{\circ}, 2 p^{21} D, 2 s^{21} S$ resonances of $\mathrm{He}$ (in $\mathrm{eV}$ ) from optimization of Eq. (5), using correlated wave functions.

\begin{tabular}{lll}
\hline \hline & \multicolumn{2}{c}{ Width (eV) } \\
Resonance & This work & Reference 7 \\
\hline $2 s 2 p{ }^{1} P^{\circ}$ & 0.041 & 0.0363 \\
$2 p^{2}{ }^{1} D$ & 0.0824 & 0.0789 \\
$2 s^{2}{ }^{1} S$ & 0.119 & 0.125 \\
\hline
\end{tabular}


TABLE II. Variation of the width of the resonance $2 p^{2}{ }^{1} D$ as a function of the parameter $d$ [Eq (4)] in the region of parameter space where the constraining Eq. (5) is satisfied best. The other parameters $K, \mu, \zeta$, and the rotation angle $\theta$ are kept constant. The optimum value of the width is that for which $\left|\left(b^{2} / a^{2}\right)-(\Gamma /|K|)\right|$ goes through an absolute minimum.

\begin{tabular}{cccc}
\hline \hline$d$ & $\left|\frac{b^{2}}{a^{2}}-\frac{\Gamma}{|K|}\right|$ & $\begin{array}{c}\text { From the complex } \\
\text { eigenvalue of the } \\
\text { diagonalized matrix } \\
\text { Equation (5) }\end{array}$ & $\begin{array}{c}\text { Width }(\mathrm{eV}) \\
\operatorname{Im}\langle H(\rho)\rangle=-\operatorname{Im}\langle T(\rho)\rangle \\
\text { (complex kinetic energy) }\end{array}$ \\
\hline 1.390 & $1250 \times 10^{-5}$ & 0.0881 & 0.0893 \\
1.395 & $590 \times 10^{-5}$ & 0.0852 & 0.0858 \\
1.400 & $62 \times 10^{-5}$ & 0.0824 & 0.0824 \\
1.405 & $300 \times 10^{-5}$ & 0.0795 & 0.0790 \\
1.410 & $1320 \times 10^{-5}$ & 0.0769 & 0.0757 \\
\hline \hline
\end{tabular}

These orbitals were used in constructing the $2 p^{21} D$ and $2 s^{2}{ }^{1} S$ configurations as well.

In addition to Eq. (5), the complex virial theorem, ${ }^{6}$

$$
\operatorname{Im}\langle H(\rho)\rangle=-\operatorname{Im}\langle T(\rho)\rangle
$$

(complex kinetic energy), was also employed as a constraining equation.

Table I presents our results for the widths which are obtained from application of Eq. (5), and those of Ref. 7 which are obtained using large wave functions and the golden-rule formula. Comparison shows that the new approach is a useful alternative to the standard theory and computation of resonance states in two electron atoms. Furthermore, and more importantly, it allows for considerable optimism regarding the treatment of Auger partial and total widths in large atoms using complex coordinates, since, to a good approximation, these will be given from the independent optimization of the asymptotic pair functions. $^{2}$

Table II presents a one-dimensional optimization of the $\mathrm{He} 2 p^{2}{ }^{1} D$ state, where Eq. (5) is finally minimized with respect to the parameter $d$ while all the other parameters are kept fixed.

\section{SUMMARY}

This report contains the first results of computations which use complex, correlated wave functions and the variational principle expressed by Eq. (5). We chose three resonances of $\mathrm{He}$ where the correlation effects are different. The ${ }^{1} P{ }^{\circ}$ state is described well by the Hartree-Fock function, as has already been pointed out. ${ }^{2,3}$ The ${ }^{1} D$ state has significant $2 p^{2} \rightarrow 2 s v_{d}$ hole-filling correlation, ${ }^{8}$ while the $2 s^{2}{ }^{1} S$ configuration mixes mainly with the $2 p^{2}$ and $2 s v_{s}$ vectors.

We found that the theory which we have proposed, regarding the choice of basis functions and variational optimization, works well in the case of He. Given the fact that $N$-electron resonances can be analyzed, to a good approximation, in terms of asymptotic pair functions and corresponding partial widths, ${ }^{2,3}$ we expect that this approach will be useful in treating manyelectron autoionizing states accurately and efficiently.

\section{ACKNOWLEDGMENTS}

We thank Dr. Y. Komninos and Mr. A. Aspromallis for useful conversations on aspects of the present computations.
${ }^{1}$ B. R. Junker, in Advances in Atomic and Molecular Physics, edited by D. R. Bates and B. Bederson (Academic, New York, 1982), Vol. 18

${ }^{2}$ C. A. Nicolaides, Y. Komninos, and Th. Mercouris, Int. J. Quantum Chem. S15, 355 (1981).

${ }^{3}$ C. A. Nicolaides and D. R. Beck, Int. J. Quantum Chem. 14, 457 (1978); C. A. Nicolaides, Phys. Rev. A $\underline{6}, 2078$ (1972).
${ }^{4}$ Y Komninos and C. A. Nicolaides, Chem. Phys. Lett. 78 347 (1981).

${ }^{5}$ C. A. Nicolaides, Y. Komninos, and Th. Mercouris, Phys. Lett. 84A, 421 (1981).

${ }^{6} \mathrm{E}$. Brändas and P. Froelich, Phys. Rev. A 16, 2207 (1977)

${ }^{7}$ A. K. Bhatia, and A. Temkin, Phys. Rev. A 11, 2018 (1975

${ }^{8}$ D. R. Beck and C. A. Nicolaides, Int. J. Quantum Chem. S14, 323 (1980); Phys. Rev. A 26, 857 (1982). 